\title{
Proteomic and bioinformatic analysis of differentially expressed proteins in denervated skeletal muscle
}

\author{
HUALIN SUN ${ }^{1,2}$, JIAYING QIU ${ }^{2}$, YANFEI CHEN ${ }^{2}$, MIAOMEI YU $^{2}$, FEI DING ${ }^{2}$ and XIAOSONG GU XI,2 $^{1,2}$ \\ ${ }^{1}$ School of Biology and Basic Medical Sciences, Soochow University, Suzhou, Jiangsu 215123; \\ ${ }^{2}$ Jiangsu Key Laboratory of Neuroregeneration, Nantong University, Nantong, Jiangsu 226001, P.R. China
}

Received November 28, 2013; Accepted April 3, 2014

DOI: $10.3892 /$ ijmm.2014.1737

\begin{abstract}
The aim of this study was to improve our understanding and the current treatment of denervation-induced skeletal muscle atrophy. We used isobaric tags for relative and absolute quantification (iTRAQ) coupled with two-dimensional liquid chromatography-tandem mass spectrometry (2DLC-MS/MS) to identify the differentially expressed proteins in the tibialis anterior (TA) muscle of rats at 1 and 4 weeks following sciatic nerve transection. A total of 110 proteins was differentially expressed and was further classified using terms from the Gene Ontology (GO) and Kyoto Encyclopedia of Genes and Genomes (KEGG) databases to unravel their molecular functions. Among the differentially expressed metabolic enzymes involved in glycolysis, Krebs cycle and oxidative phosphorylation, $\alpha$ - and $\beta$-enolase displayed an increased and decreased expression, respectively, which was further validated by western blot analysis and immunohistochemistry. These findings suggest that the enolase isozymic switch during denervation-induced muscle atrophy is the reverse of that occurring during muscle maturation. Notably, protein-protein interaction analysis using the STRING database indicated that the protein expression of tumor necrosis factor receptor-associated factor- 6 (TRAF6), muscle ring-finger protein 1 (MuRF1) and muscle atrophy F-box (MAFBx) was also upregulated during denervation-induced skeletal muscle atrophy, which was confirmed by western blot analysis. TRAF6 knockdown experiments in L6 myotubes suggested that the decreased expression of TRAF6 attenuated glucocorticoid-induced myotube atrophy. Therefore, we hypothesized that the upregulation of TRAF6 may be involved in the development of denervation-induced muscle atrophy, at least in part, by regulating the expression of MAFBx and MuRF1 proteins. The data from the present
\end{abstract}

Correspondence to: Professor Xiaosong Gu, Jiangsu Key Laboratory of Neuroregeneration, Nantong University, 19 Qixiu Road, Nantong, Jiangsu 226001, P.R. China

E-mail: nervegu@ntu.edu.cn

Key words: proteomics, bioinformatics, skeletal muscle atrophy, denervation, enolase, tumor necrosis factor receptor-associated factor-6 study provide valuable insight into the molecular mechanisms regulating denervation-induced muscle atrophy.

\section{Introduction}

Skeletal muscle atrophy is a complex biochemical process occurring under various pathophysiological conditions in adult animals, such as disuse (e.g., immobilization, denervation, muscle unloading), starvation, aging and in syndromes, such as cachexia (1). It is important to understand the molecular mechanisms underlying skeletal muscle atrophy and to develop effective strategies to delay its onset (2-4). Denervation-induced muscle atrophy has received attention as it is commonly encountered in clinical practice and is very likely to cause extreme adverse effects $(5,6)$. A number of factors that contribute to denervation-induced muscle atrophy have been identified, including neuromuscular alterations, altered protein synthesis and degradation, and apoptosis-induced muscle fiber loss (7-10). However, previous studies have mainly focused on single gene and/or protein changes potentially linked to skeletal muscle atrophy. Therefore, a global investigation of the protein expression changes may help to decipher the molecular basis of denervation-induced skeletal muscle atrophy.

Proteomics is a well-established approach for simultaneously detecting the expression of a high number of proteins in biological samples. Among the different proteomic techniques, isobaric tags for relative and absolute quantification (iTRAQ) labeling, albeit initially developed on the basis of traditional two-dimensional electrophoresis (2-DE), is particularly suitable and has proven to far surpass 2-DE in sample coverage and protein separation efficiency (11). iTRAQ coupled with two-dimensional liquid chromatography-tandem mass spectrometry (2D LC-MS/MS) represents a state-of-the art tool, extensively used to identify and quantify differential proteomes.

In this study, we first examined the protein expression profile in denervated tibialis anterior (TA) muscle following sciatic nerve transection in rats, and then classified the differentially expressed proteins using Gene Ontology (GO) functional annotation and the Kyoto Encyclopedia of Genes and Genomes (KEGG) pathway terms, so as to investigate the functional implications of differential expression in TA muscle during denervation-induced atrophy. Additional protein-protein interaction analysis revealed that the tumor necrosis factor receptor-associated factor-6 (TRAF6) protein 
was also differentially expressed in denervated TA muscle, although this protein was not detected in our proteomics analysis, possibly due to its low abundance. TRAF6 knockdown experiments provided further evidence of the biological significance of TRAF6 in skeletal muscle atrophy.

\section{Materials and methods}

Animals and surgical procedures. Adult female Sprague-Dawley (SD) rats, weighing 180-220 g, were provided by the Experimental Animal Center of Nantong University. The rats were randomly divided into 2 groups, subsequently subjected to operation, and 1 control group (10 rats in each group). Animal handling procedures followed the Institutional Animal Care Guidelines of Nantong University and were approved by the Administration Committee of Experimental Animals, Jiangsu Province, China. The rats were subjected to sciatic nerve transection (operated groups) or sham operation (control group), as previously described (12). At 1 and 4 weeks following the operation, the TA muscles were rapidly dissected from the operated side of the animals and immediately immersed in liquid nitrogen prior to use. TA muscles were also harvested from animals of the control group.

Protein sample preparation and $i T R A Q$ labeling. Protein samples were extracted from the harvested muscles and quantified using the Protein Assay kit (Bio-Rad, Hercules, CA, USA). iTRAQ labeling of the protein samples was performed as previously described (12). Briefly, $100 \mu \mathrm{g}$ of each protein sample, obtained by acetone precipitation, was dissolved in $20 \mu \mathrm{l}$ of dissolution buffer and sequentially reduced, alkylated and digested, followed by labeling with the iTRAQ tags 114 , 115 and 116, and pooling for further analysis.

The mixed iTRAQ-labeled sample was resuspended in buffer $\mathrm{A}\left(10 \mathrm{mM} \mathrm{KH}_{2} \mathrm{PO}_{4}\right.$ in $25 \% \mathrm{v} / \mathrm{v}$ acetonitrile at $\left.\mathrm{pH} 2.7\right)$ and fractionated using an off-line strong cation exchange column on a 1100 HPLC system (Agilent Technologies, Waldbronn, Germany). Gradient elution was performed from $0 \%$ buffer B $\left(10 \mathrm{mM} \mathrm{KH} \mathrm{KO}_{4}\right.$ in $25 \% \mathrm{v} / \mathrm{v}$ acetonitrile/350 mM KCL at $\mathrm{pH} 2.7$ ) to $25 \%$ buffer B for $30 \mathrm{~min}$, and then from $25 \%$ buffer $\mathrm{B}$ to $100 \%$ buffer B for $20 \mathrm{~min}$. The fractions were collected at 2-min intervals, and desalted on Vivapure ${ }^{\circledR}$ C18 Micro spin columns (Sartorius Stedim Biotech, Gottingen, Germany), and vacuum-dried prior to LC/MS/MS analysis. All reagents used in these procedures were purchased from Applied Biosystems (Foster City, CA, USA).

$2 D$ LC-MS/MS. Online 2D nano LC-MS/MS analysis was performed as previously described (12). Briefly, the peptides in each fraction were resuspended in $20 \mu \mathrm{l}$ solvent $\mathrm{A}$ (water with $0.1 \%$ formic acid), separated by nano LC, and analyzed by on-line electrospray tandem mass spectrometry using the LTQ Orbitrap XL mass spectrometer (Thermo Electron Corp., Bremen, Germany). An 18- $\mu 1$ peptide sample was loaded for $5 \mathrm{~min}$, with a flow of $20 \mu \mathrm{l} / \mathrm{min}$, onto the peptide column Captrap (Michrom BioResources, Auburn, CA, USA), and subsequently eluted with a 3-step linear gradient, starting from 5\% solvent B (acetonitrile with $0.1 \%$ formic acid) to $45 \%$ solvent B for $70 \mathrm{~min}$, increased to $80 \%$ solvent B for $1 \mathrm{~min}$, and then holding on $80 \%$ solvent B for $4 \mathrm{~min}$. The electrospray voltage of $1.9 \mathrm{kV}$ vs. the inlet of the mass spectrometer was used.
A LTQ Orbitrap XL mass spectrometer was operated in the data-dependent mode to switch automatically between MS and MS/MS acquisition. Survey full-scan MS spectra (m/z 400-2,000) were acquired with a mass resolution of 60,000 at $\mathrm{m} / \mathrm{z} 400$, followed by MS/MS of the 4 most intense peptide ions. The dissociation mode was higher energy C-trap dissociation (HCD), under which iTRAQ-labeled peptides fragmented to produce reporter ions at 114.1, 115.1 and 116.1. Fragment ions of the peptides were simultaneously produced, and sequencing of the labeled peptides allowed the identification of the corresponding proteins. Dynamic exclusion was used with 2 repeat counts (10-sec repeat duration), and the $\mathrm{m} / \mathrm{z}$ values triggering MS/MS were placed on an exclusion list for $120 \mathrm{sec}$. For MS/ MS, precursor ions were activated using $40 \%$ normalized collision energy and an activation time of $30 \mathrm{msec}$. The peak intensity of the 3 iTRAQ reporter ions reflected the relative abundance of the peptides and thereby, proteins, in the samples.

Protein identification and quantification. The MS raw data were analyzed as previously described (12). Briefly, MS/MS spectra were compared to rat data from the Swiss-Prot database (Release 2010_04) using the SEQUEST software v.28 (revision 12; Thermo Electron Corp.). The search parameters were set as follows: trypsin (KR) cleavage with 2 miscleavages allowed; carbamidomethylation of cysteine residues as fixed modification; iTRAQ modification of peptide N-termini, methionine oxidation, iTRAQ modification of lysine residues and $\mathrm{N}$-terminal acetylation as variable modifications; peptide mass tolerance $20 \mathrm{ppm}$, and fragment ion tolerance 0.05 Da. Protein identification results were evaluated using the Trans Proteomic Pipeline (TPP) set of tools (revision 4.2), with quantification of iTRAQ reporter ion intensities performed using the Libra tool.

For the selection of differentially expressed proteins, we considered the following criteria, as previously described (13): i) proteins containing at least 2 unique high-scoring peptides; and ii) proteins with a median ratio above 2 or below 0.5 ; and iii) $>95 \%$ confidence level in each comparison.

Bioinformatic analysis. The differentially expressed proteins were mapped to the appropriate GO database to calculate the number of genes at each node, and were classified according to molecular function. The differentially expressed proteins were further classified into the KEGG molecular pathway (http://www.genome.jp/kegg/pathway.html) to explore specific biological pathways affected by skeletal muscle atrophy. In addition, predicted protein-protein interactions for the list of differentially expressed proteins and the resulting network were retrieved and constructed using the STRING database version 9.0 (http://string-db.org) (14).

Western blot analysis. Western blot analysis was used to confirm the expression of selected proteins as previously described (15). Briefly, muscle protein samples were homogenized in RIPA buffer, separated by 1D electrophoresis and electroblotted onto a polyvinylidene fluoride (PVDF) membrane. The membrane was blocked with $5 \%$ non-fat dry milk in Tris-buffered saline (TBS) for $1 \mathrm{~h}$ at room temperature, followed by incubation with primary polyclonal antibodies: mouse anti- $\beta$-enolase (1:1,000; BD Biosciences, San Diego, CA, USA) and rabbit anti$\alpha$-enolase (1:500; AB Biotec, Stockholm, Sweden) in TBST 
(10 mM Tris-HCl, pH 7.5, $150 \mathrm{mM} \mathrm{NaCl}$ and $0.1 \%$ Tween-20) supplemented with $5 \%$ milk overnight at $4^{\circ} \mathrm{C}$. After washing in TBST, the membrane was incubated with HRP-conjugated goat anti-rabbit/mouse IgG polyclonal antibody (AB Biotec) for $60 \mathrm{~min}$. Following TBST washes, immunoprobed proteins were visualized using the enhanced chemiluminescence method: Chemiluminescent solution luminol and hydrogen peroxide were provided with the ECL luminescence kit (Pierce Corp., Rockford, IL, USA). HRP catalyzes the reaction of luminol with hydrogen peroxide to generate a peroxide. The peroxide is unstable and easy to decompose to form a luminescent electron excitation energy intermediates, which will produce fluorescence, when the electron excitation energy intermediates return from the excited state to the ground state.

Immunohistochemistry. Immunohistochemical analysis was performed as described in a previous study (15). Briefly, the TA muscle was dissected, post-fixed, dehydrated, and sectioned (8- $\mu$ m-thick sections) using a cryostat; the sections were thawmounted onto poly-L-lysine-coated slides and stored at $-20^{\circ} \mathrm{C}$ prior to immunostaining. The slides were washed in phosphatebuffered saline (PBS) for $10 \mathrm{~min}$ at room temperature, blocked, and then incubated overnight at $4^{\circ} \mathrm{C}$ with primary antibodies: mouse anti- $\beta$-enolase antibody and rabbit anti- $\alpha$-enolase antibody (both at 1:100). After washing with PBS, the slides were incubated at $4^{\circ} \mathrm{C}$ for $24 \mathrm{~h}$ with secondary goat antibodies labeled with fluorescein isothiocyanate 1 (FITC): anti-mouse IgG-FITC (1:100; Santa Cruz Biotechnology, Inc., Santa Cruz, CA, USA) and anti-rabbit IgG-FITC (1:200; Abcam, Cambridge, MA, USA). The slides were washed 3 times in PBS, coverslipped and visualized under a DMR fluorescent microscope (Leica Microsystems, Wetzlar, Germany).

Cell culture and small interfering RNA (siRNA) transfection. The cells were cultured as previously described (16). Briefly, L6 skeletal muscle cells were grown and maintained in high-glucose Dulbecco's modified Eagle's medium (DMEM) supplemented with $10 \%$ fetal bovine serum (FBS), $100 \mathrm{U} / \mathrm{ml}$ penicillin and $100 \mu \mathrm{g} / \mathrm{ml}$ streptomycin in a $10 \% \mathrm{CO}_{2}$ humidified atmosphere at $37^{\circ} \mathrm{C}$. The cells grown in culture flasks up to approximately $80 \%$ confluency were trypsinized and seeded into a 6-well culture plate for incubation in DMEM containing $10 \%$ FBS, until they reached approximately $90 \%$ confluence. Following replacement of the medium with DMEM containing $2 \%$ horse serum, the cells were induced to differentiate until $>90 \%$ had differentiated into myotubes. The resulting L6 myotubes were treated with $100 \mathrm{nM}$ dexamethasone in $0.1 \%$ ethanol for $48 \mathrm{~h}$. Dexamathasone is a glucocorticoid that induces myotube atrophy (16).

TRAF6 siRNA oligonucleotides targeting rat TRAF6, and control oligonucleotides (TRAF6 siRNA negative control) were purchased from RiboBio Co., Ltd. (Guangzhou, China). Cells were transfected using riboFect ${ }^{\mathrm{TM}} \mathrm{CP}$ reagent (RiboBio Co., Ltd.) according to the manufacturer's instructions. L6 myotubes were transfected with $100 \mathrm{nM}$ TRAF6 siRNA or $100 \mathrm{nM}$ negative control siRNA. Six hours later, the medium was replaced with differentiation medium. L6 myotubes were treated with $100 \mathrm{nM}$ dexamathasone in $0.1 \%$ ethanol for $48 \mathrm{~h}$ and collected for RNA preparation. For myotube size quantification, the transfected L6 myotubes were fixed after $48 \mathrm{~h}$ of dexamatheasone treatment. Myotube cultures were photographed under a phase contrast microscope (Leica Microsystems). The diameters were measured in a total of 60 myotubes from at least 6 random fields using Image-Pro Plus software (Media Cybernetics, Silver Springs, MD, USA).

Quantitative reverse-transcription PCR ( $q R T-P C R)$. Total RNA was extracted from the L6 myotubes, and reverse transcription was performed using Oligo(dT) primers (Shanghai Sangon Biotechnology Corp., Shanghai, China). cDNA was synthesized using an iScript cDNA Synthesis kit (Bio-Rad) following the manufacturer's instructions, and stored at $-20^{\circ} \mathrm{C}$ prior to use. All primers were purchased from Generay Biotech Co., Ltd. (Shanghai, China). The primers used in this study were as folows: TRAF6 forward, GGCA TTTACATTTGGAAGATTGGC and reverse, AGGGAAATG TAGTTTGCACAGCG; muscle ring-finger protein 1 (MuRF1) forward, GGTGCCTACTTGCTCCTTGTGC and reverse, CTGTTTTCCTTGGTCACTCGGC; muscle atrophy F-box (MAFbx) forward, GATCTTGTCTGACAAAGGGCAGC and reverse, GGGTGAAAGTGAGACGGAGCAG and GAPDH forward, CAACGGGAAACCCATCACCA and reverse, ACG CCAGTAGACTCCACGACAT. The PCR reactions were performed on the Applied Biosystems 7500 real-time PCR system using the iTaq Fast SYBR-Green Supermix (Bio-Rad) following the manufacturer's instructions. The cycle threshold $(\mathrm{Ct})$ values, corresponding to the PCR cycle number at which fluorescence emission reached a threshold above baseline emission, were determined. mRNA expression levels were then calculated using the $2^{-\Delta \Delta \mathrm{Ct}}$ method, as described in a previous study (16). GAPDH served as an internal control.

Statistical analysis. All data are expressed as the means \pm SD. One-way ANOVA was used to compare differences between groups. All statistical analyses were conducted with the Stata 7.0 software package (StataCorp LP, College Station, TX, USA). Values of $\mathrm{p}<0.05$ were considered to indicate statistically significant differences.

\section{Results}

Screening of differentially expressed proteins and functional analysis

ITRAQ-based proteomic analysis. A total of 110 proteins were identified as differentially expressed (with criteria: $p<0.05$ and fold change of $>2.0$ ) in denervated TA muscle at 1 and 4 weeks following sciatic nerve transection. The 110 proteins are listed in Table I, and their expression levels relative to the control are displayed in a heatmap graphic (Fig. 1).

GO functional annotation. The 110 differentially expressed proteins were grouped into 11 classes according to their molecular function based on GO terms (Table I). The highest number of proteins was classified as metabolic enzymes, followed by structural proteins, signaling molecules, chaperones, extracellular matrix proteins and ubiquitin proteasome pathway-related proteins.

$K E G G$ pathway identification. The 110 differentially expressed proteins were involved in a number of distinct pathways, such as glycolysis, Krebs (tricarboxylic acid/citrate) cycle, proteasome and MAPK signaling. The heatmap displaying the 
Table I. List of differentially expressed proteins in denervated tibialis anterior (TA) muscle.

\begin{tabular}{|c|c|c|c|}
\hline $\begin{array}{l}\text { Functional category: } \\
\text { gene name }\end{array}$ & $115 / 114$ & $116 / 114$ & Predicted molecular function/protein name \\
\hline \multicolumn{4}{|l|}{ Metabolic enzymes } \\
\hline ACADL_RAT & 0.756 & 2.180 & Long-chain specific acyl-CoA dehydrogenase, mitochondrial \\
\hline CAH3_RAT & 0.300 & 4.451 & Carbonic anhydrase 3 \\
\hline CYB5_RAT & 0.692 & 2.077 & Cytochrome b5 \\
\hline D3D2_RAT & 0.444 & 1.778 & 3,2-Trans-enoyl-CoA isomerase, mitochondrial \\
\hline ENOA_RAT & 0.725 & 2.102 & Alpha-enolase \\
\hline ENOB_RAT & 1.236 & 0.302 & Beta-enolase \\
\hline ESTD_RAT & 0.923 & 2.538 & S-formylglutathione hydrolase \\
\hline FPPS_RAT & 1.028 & 2.355 & Farnesyl pyrophosphate synthetase \\
\hline G3P_RAT & 1.445 & 0.429 & Glyceraldehyde-3-phosphate dehydrogenase \\
\hline GSTM2_RAT & 0.316 & 1.158 & Glutathione S-transferase Mu 2 \\
\hline HADH_RAT & 1.820 & 0.261 & Hydroxyacyl-coenzyme A dehydrogenase, mitochondrial \\
\hline K6PF_RAT & 2.512 & 0.288 & 6-Phosphofructokinase, muscle type \\
\hline KCC2A_RAT & 0.300 & 0.425 & Calcium/calmodulin-dependent protein kinase type II alpha chain \\
\hline KCC2G_RAT & 0.923 & 2.000 & Calcium/calmodulin-dependent protein kinase type II gamma chain \\
\hline KCRB_RAT & 1.000 & 2.286 & Creatine kinase B-type \\
\hline KCRM_RAT & 1.271 & 0.373 & Creatine kinase M-type \\
\hline KCRS_RAT & 0.600 & 0.425 & Creatine kinase, sarcomeric mitochondrial \\
\hline NDKB_RAT & 0.778 & 3.444 & Nucleoside diphosphate kinase B \\
\hline PDIA1_RAT & 0.824 & 2.015 & Protein disulfide-isomerase \\
\hline PDIA6_RAT & 1.400 & 3.400 & Protein disulfide-isomerase A6 \\
\hline PPIA_RAT & 2.000 & 10.000 & Peptidyl-prolyl cis-trans isomerase A \\
\hline PPIB_RAT & 0.400 & 3.300 & Peptidyl-prolyl cis-trans isomerase B \\
\hline PRDX1_RAT & 1.375 & 4.375 & Peroxiredoxin-1 \\
\hline PYGB_RAT & 1.355 & 0.425 & Glycogen phosphorylase, brain form (fragment) \\
\hline PYGM_RAT & 0.895 & 0.354 & Glycogen phosphorylase, muscle form \\
\hline ATP5H_RAT & 1.412 & 2.753 & ATP synthase subunit $d$, mitochondrial \\
\hline IDH3B_RAT & 0.488 & 0.847 & Isocitrate dehydrogenase $\left(\mathrm{NAD}^{+}\right)$subunit beta, mitochondrial \\
\hline NB5R3_RAT & 0.337 & 2.228 & NADH-cytochrome b5 reductase 3 \\
\hline \multicolumn{4}{|l|}{ Structural proteins } \\
\hline ACTN1_RAT & 0.904 & 2.604 & Alpha-actinin-1 \\
\hline CAP1_RAT & 0.800 & 2.467 & Adenylyl cyclase-associated protein 1 \\
\hline CAP2_RAT & 0.462 & 0.846 & Adenylyl cyclase-associated protein 2 \\
\hline COF1_RAT & 0.909 & 3.182 & Cofilin-1 \\
\hline CSRP3_RAT & 1.312 & 6.750 & Cysteine and glycine-rich protein 3 \\
\hline DESM_RAT & 0.055 & 0.575 & Desmin \\
\hline FHL1_RAT & 0.667 & 2.200 & Four and a half LIM domains protein 1 \\
\hline LMNA_RAT & 0.750 & 3.875 & Lamin-A \\
\hline MLE1_RAT & 1.047 & 0.394 & Myosin light chain 1, skeletal muscle isoform \\
\hline MLRS_RAT & 1.038 & 0.421 & Myosin regulatory light chain 2 , skeletal muscle isoform \\
\hline MOES_RAT & 1.019 & 2.443 & Moesin \\
\hline MYH8_RAT & 1.000 & 2.780 & Myosin-8 (fragment) \\
\hline PDLI7_RAT & 1.917 & 2.750 & PDZ and LIM domain protein 7 \\
\hline PLEC1_RAT & 0.786 & 2.214 & Plectin-1 \\
\hline TAGL2_RAT & 0.658 & 2.225 & Transgelin-2 \\
\hline TBA1A_RAT & 1.625 & 5.125 & Tubulin alpha- $1 \mathrm{~A}$ chain \\
\hline TBA4A_RAT & 0.917 & 2.917 & Tubulin alpha-4A chain \\
\hline TNNT3_RAT & 1.019 & 0.360 & Troponin $\mathrm{T}$, fast skeletal muscle \\
\hline TPM4_RAT & 0.222 & 0.815 & Tropomyosin alpha-4 chain \\
\hline \multicolumn{4}{|l|}{ Signaling molecules } \\
\hline ADT1_RAT & 2.070 & 1.038 & ADP/ATP translocase 1 \\
\hline ARRS_RAT & 87.902 & 74.473 & S-arrestin \\
\hline AT1A1_RAT & 1.000 & 2.231 & Sodium/potassium-transporting ATPase subunit alpha-1 \\
\hline AT1A2_RAT & 0.367 & 0.600 & Sodium/potassium-transporting ATPase subunit alpha-2 \\
\hline AT2A1_RAT & 2.300 & 2.502 & Sarcoplasmic/endoplasmic reticulum calcium ATPase 1 \\
\hline
\end{tabular}


Table I. (Continued).

Functional category:

Signaling molecules

CALM_RAT
CASQ1_RAT
GDIR1_RAT
GELS_RAT
LEG1_RAT
MPCP_RAT
PEBP1_RAT
RAB14_RAT
SPA3K_RAT
SYUG_RAT
VDAC2_RAT

Chaperones

1433Z_RAT
CRYAB_RAT
GRP78_RAT
HSP71_RAT
HSPB2_RAT
HSPB7_RAT

Extracellular matrix proteins

CO1A1_RAT
CO1A2_RAT
LUM_RAT
PRELP_RAT

Ubiquitin proteasome pathway

$$
\begin{aligned}
& \text { PSA5_RAT } \\
& \text { UBE2N_RAT }
\end{aligned}
$$

Carrier proteins

$$
\begin{aligned}
& \text { ACBP_RAT } \\
& \text { ALBU_RAT } \\
& \text { HBB1_RAT } \\
& \text { HEMO_RAT } \\
& \text { MYG_RAT } \\
& \text { TRFE_RAT }
\end{aligned}
$$

Ribosomal proteins and histones

$\begin{array}{llr}\text { H10_RAT } & 0.738 & 2.150 \\ \text { H2A1_RAT } & 1.500 & 11.500 \\ \text { H2A3_RAT } & 0.492 & 2.014 \\ \text { H2AJ_RAT } & 1.750 & 7.750 \\ \text { H2AY_RAT } & 0.286 & 0.750 \\ \text { H2AZ_RAT } & 1.000 & 3.273 \\ \text { H2B1A_RAT } & 0.946 & 2.630 \\ \text { H33_RAT } & 1.600 & 7.600 \\ \text { RL10_RAT } & 0.533 & 2.800 \\ \text { RL10A_RAT } & 1.000 & 3.100 \\ \text { RL23A_RAT } & 0.667 & 2.467 \\ \text { RS14_RAT } & 0.424 & 0.727 \\ \text { RS23_RAT } & 0.571 & 3.143 \\ \text { RS25_RAT } & 0.500 & 4.200 \\ \text { Membrane trafficking proteins } & & \\ \text { NSF1C_RAT } & 1.600 & 3.500 \\ \text { RTN4_RAT } & 0.104 & 0.991 \\ \text { TTHY_RAT } & 2.377 & 1.600 \\ \text { VAPA_RAT } & 0.700 & 3.200\end{array}$

\author{
Calmodulin \\ Calsequestrin-1 (fragment) \\ Rho GDP-dissociation inhibitor 1 \\ Gelsolin \\ Galectin-1 \\ Phosphate carrier protein, mitochondrial \\ Phosphatidylethanolamine-binding protein 1 \\ Ras-related protein Rab-14 \\ Serine protease inhibitor A3K \\ Gamma-synuclein \\ Voltage-dependent anion-selective channel protein 2
}

14-3-3 protein zeta/delta

Alpha-crystallin B chain

$78 \mathrm{kDa}$ glucose-regulated protein

Heat shock $70 \mathrm{kDa}$ protein $1 \mathrm{~A} / 1 \mathrm{~B}$

Heat shock protein beta- 2

Heat shock protein beta-7 (fragment)

Collagen alpha-1 (I) chain

Collagen alpha-2 (I) chain

Lumican

Prolargin

Proteasome subunit alpha type-5

Ubiquitin-conjugating enzyme E2 N

Acyl-CoA-binding protein

Serum albumin

Hemoglobin subunit beta-1

Hemopexin

Myoglobin

Serotransferrin

Histone H1.0

Histone H2A type 1

Histone H2A type 3

Histone H2A.J

Core histone macro-H2A.1

Histone H2A.Z

Histone $\mathrm{H} 2 \mathrm{~B}$ type 1-A

Histone H3.3

60S ribosomal protein L10

$60 \mathrm{~S}$ ribosomal protein $\mathrm{L} 10 \mathrm{a}$

$60 \mathrm{~S}$ ribosomal protein $\mathrm{L} 23 \mathrm{a}$

$40 \mathrm{~S}$ ribosomal protein $\mathrm{S} 14$

$40 \mathrm{~S}$ ribosomal protein $\mathrm{S} 23$

40S ribosomal protein S25

NSFL1 cofactor $\mathrm{p} 47$

Reticulon-4

Transthyretin

Vesicle-associated membrane protein-associated protein A 
Table I. (Continued).

Functional category:

gene name $\quad 115 / 114 \quad 116 / 114 \quad$ Predicted molecular function/protein name

Protein synthesis

KBTBA_RAT

Other proteins

$\begin{array}{lll}\text { ANKR1_RAT } & 0.047 & 1.213 \\ \text { CISD1_RAT } & 0.364 & 1.045 \\ \text { CO3_RAT } & 1.015 & 3.195 \\ \text { COQ9_RAT } & 3.467 & 1.294 \\ \text { CYTC_RAT } & 0.319 & 1.837 \\ \text { IGG2A_RAT } & 0.240 & 5.230 \\ \text { IGG2B_RAT } & 1.420 & 5.569 \\ \text { KACB_RAT } & 1.009 & 9.908 \\ \text { PBIP1_RAT } & 0.818 & 3.273 \\ \text { QCR8_RAT } & 0.235 & 0.333\end{array}$

Kelch repeat and BTB domain-containing protein 10

Ankyrin repeat domain-containing protein 1

CDGSH iron sulfur domain-containing protein 1

Complement C3

Ubiquinone biosynthesis protein COQ9, mitochondrial

Cystatin-C

Ig gamma-2A chain $\mathrm{C}$ region

Ig gamma-2B chain $\mathrm{C}$ region

Ig kappa chain $\mathrm{C}$ region, $\mathrm{B}$ allele

Pre-B-cell leukemia transcription factor-interacting protein 1 Cytochrome b-c1 complex subunit 8
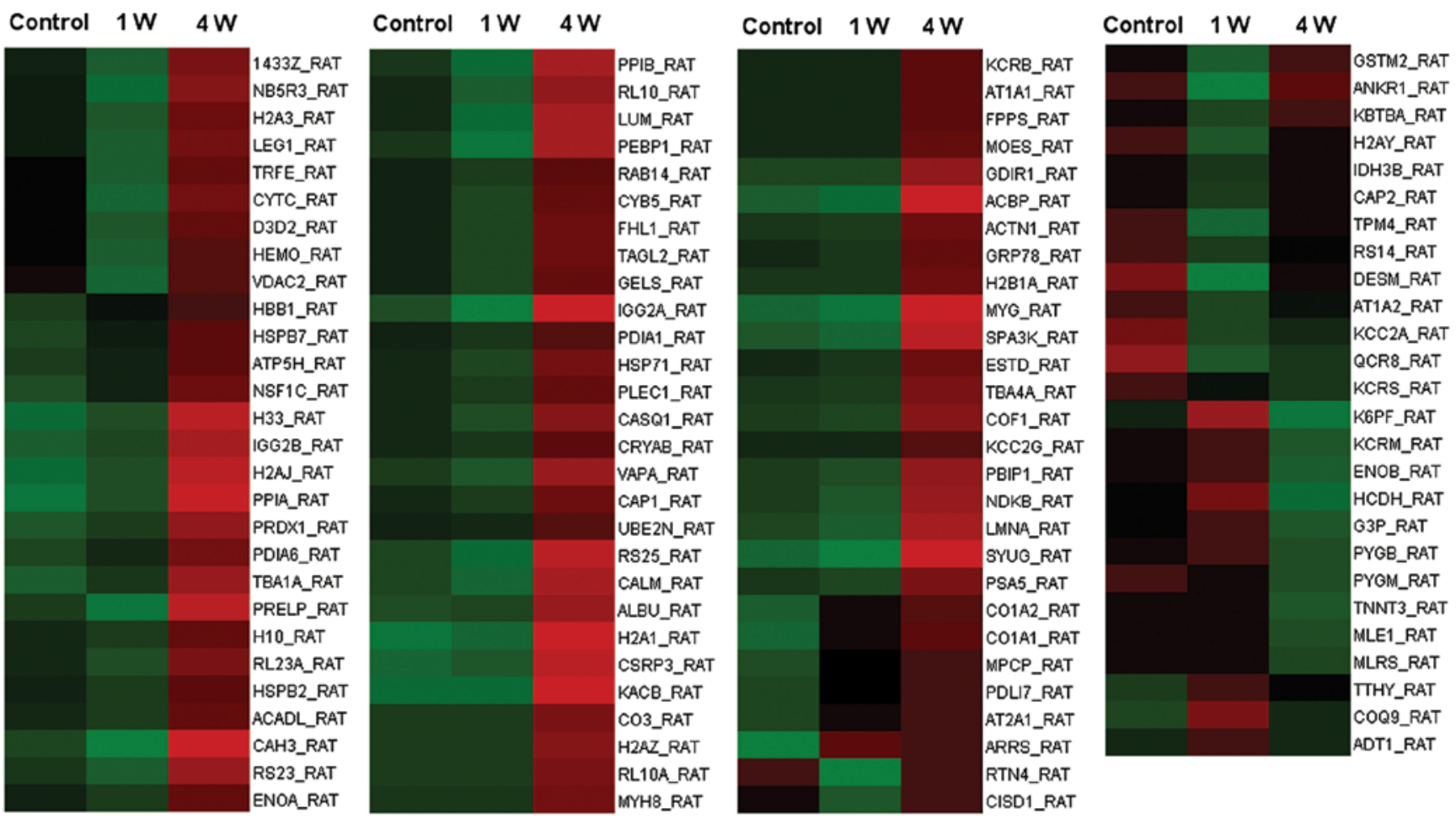

Figure 1. Heatmap displaying the expression of differentially expressed proteins identified from the proteomics analysis at 0 (control), 1 ( $1 \mathrm{~W})$ and $4 \mathrm{weeks}(4 \mathrm{~W})$ following nerve transection. The color scale illustrates the relative expression level of each protein across the 3 samples; red and green indicate higher and lower expression compared to the median expression value (black), respectively. The color intensity indicates the degree of protein up- or downregulation.

expression data for the corresponding genes (Fig. 2) shows that during denervation-induced muscle atrophy, decreasing trends are observed for the Krebs cycle and glycolysis (at 4 weeks), while proteasome and MAPK signaling pathway genes showed an increasing trend at 4 weeks. Moreover, glycolysis-related genes were the most highly expressed in all conditions.

Analysis with STRING databases. We searched for known and predicted interactions for the differentially expressed proteins identified by iTRAQ-based proteomics in the STRING protein-protein interaction database and constructed a protein-protein interaction network (Fig. 3). The network predicted an interaction between ubiquitin-conjugating enzyme E2N (UBE2N), identified as upregulated in denervated atrophic skeletal muscle from our proteomics analysis, and TRAF6, which was not detected in our study. UBE2N is required for TRAF6 activation (17). Therefore, we hypothesized that TRAF6 may also be expressed in denervated TA muscle and upregulated during denervation-induced atrophy. Furthermore, it was shown that TRAF6 activates both MuRF1 and muscle-specific ubiquitin E3-ligase atrophy gene-1/muscle 


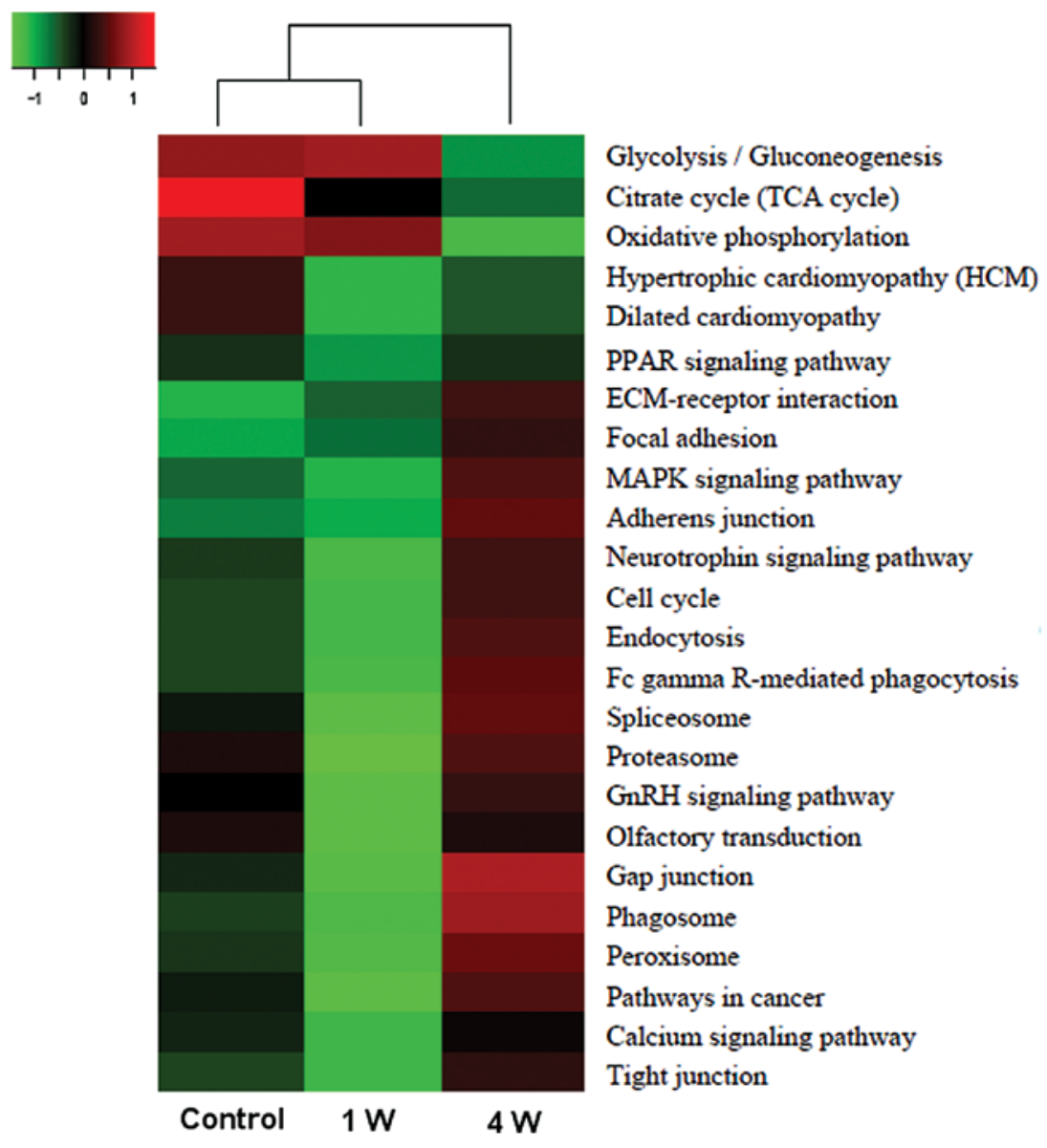

Figure 2. Heatmap cluster dendrogram, showing the relative expression level of differentially expressed proteins partitioned in different KEGG pathways at 0 (control) $1(1 \mathrm{~W})$ and 4 weeks $(4 \mathrm{~W})$ following nerve transection. Higher expression relative to median expression value (black) is denoted by red and lower expression is denoted by green color.

atrophy F-box (Atrogin-1/MAFbx) (18), which further suggests that the expression of MuRF1 and Atrogin-1/MAFbx is upregulated during the progression of muscle atrophy. Both hypotheses derived from the network analysis were confirmed by western blot analysis (see below).

Validation of selected differentially expressed proteins. To validate the results obtained by iTRAQ coupled with 2DLC-MS/MS, 2 representative glycolytic enzymes, $\alpha$ - and $\beta$-enolase, were selected for western blot and immunohistochemical analyses. In these analyses, $\alpha$ - and $\beta$-enolase were found to be gradually up- and downregulated during denervation-induced atrophy in TA muscle, respectively (Fig. 4A and B). The comparison between the western blot analysis and iTRAQ-based proteomics results for the 2 enzymes indicated that the expression change trends were consistent overall between the 2 methods, despite some deviations (Fig. 4A).

Western blot analysis was also carried out to confirm the hypothesis derived from the protein-protein interaction network. The results indicated that the protein expression of TRAF6, MAFBx and MuRF1 was significantly upregulated during denervation-induced muscle atrophy in TA muscle (Fig. 5).

Involvement of TRAF6 in myotube atrophy. Light microscopy revealed that transfection of myotubes with the siRNA targeting TRAF6 attenuated dexamethasone-induced atrophy of L6 myotubes as compared to transfection with the negative control siRNA (Fig. 6A). The diameter of L6 myotubes in which TRAF6 was knocked down was significantly larger than that of the myotubes transfected with the negative control (Fig. 6B). In addition, qRT-PCR demonstrated the dexamethasone-induced upregulation of MAFBx and MuRF1, as well as the expected downregulation of TRAF6 expression by transfection with siRNA (Fig. 6C).

\section{Discussion}

In this study, proteomic and bioinformatic analyses were performed to examined the changes in TA muscle during denervation-induced atrophy. Our findings are in agreement with results from previous global protein expression profiling studies in denervated skeletal muscle $(12,19)$, but also identified novel protein targets that may be relevant to the pathobiology of muscle atrophy.

The majority of differentially expressed proteins in denervated TA muscle identified in our study are enzymes involved in the regulation of energy metabolism, including $\alpha$ - and $\beta$-enolase, glycogen phosphorylase muscle form (PYGM), creatine kinase M-type (KCRM) and GAPDH (G3P). Crossreferencing with KEGG pathway data indicated that these 


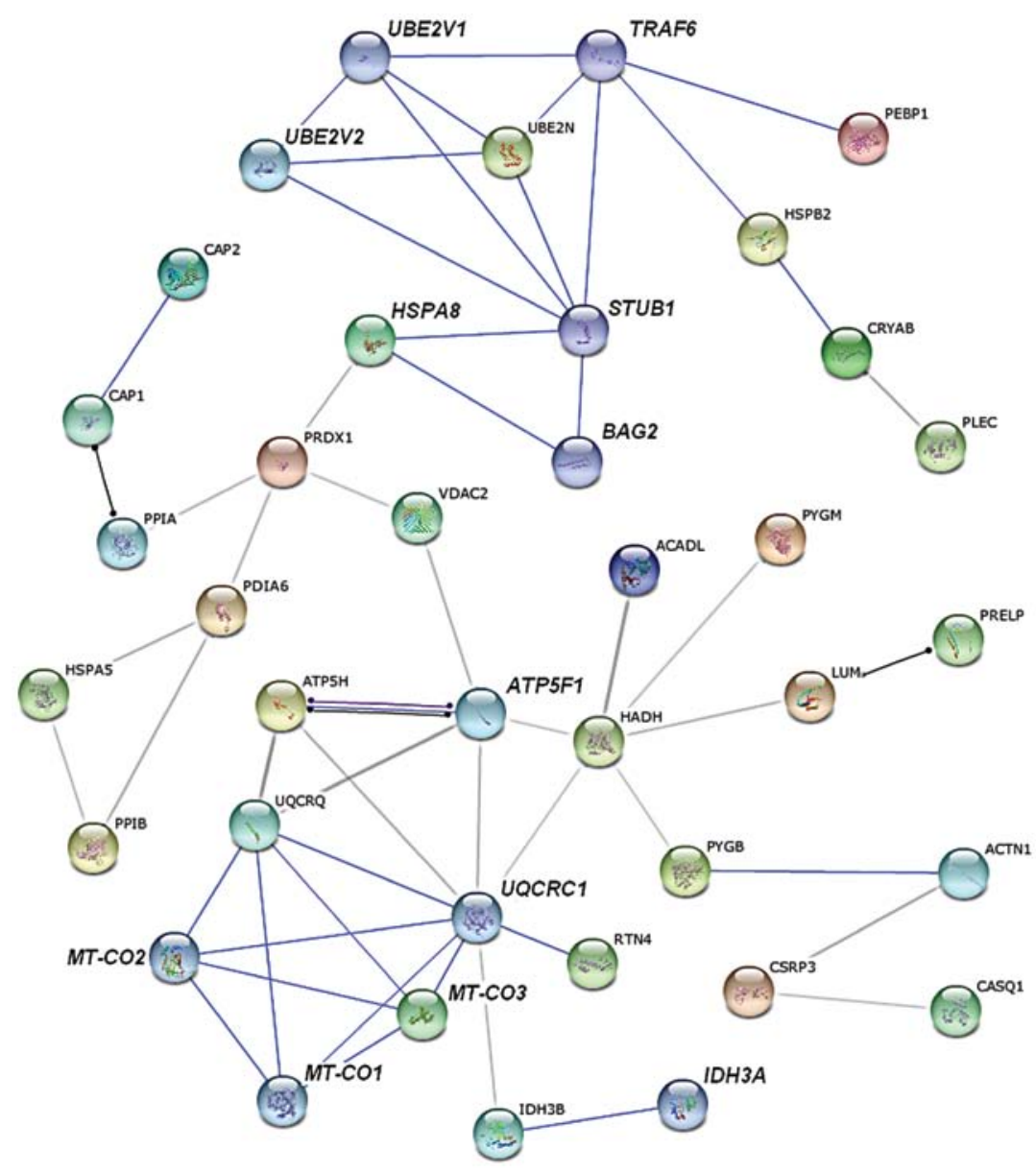

Figure 3. Protein-protein interactions for the differentially expressed proteins identified by iTRAQ-based proteomics were analyzed with the STRING software. In the network, proteins are represented as nodes. Proteins labeled in bold italics are those undetected by the proteomic study. Colors of the lines connecting the nodes represent different evidence types for protein linkage.

A

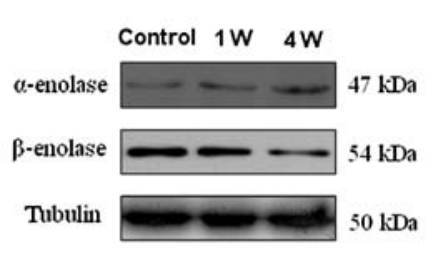

B
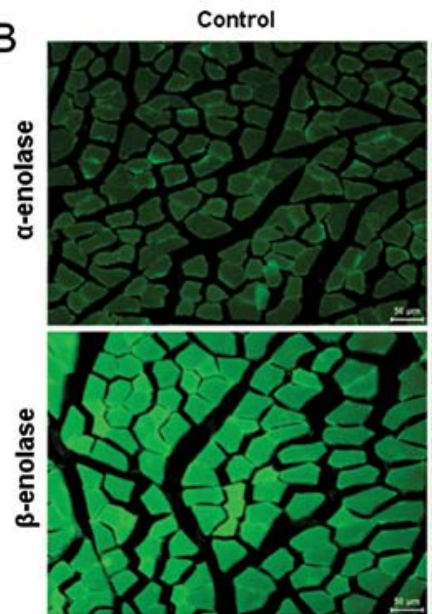
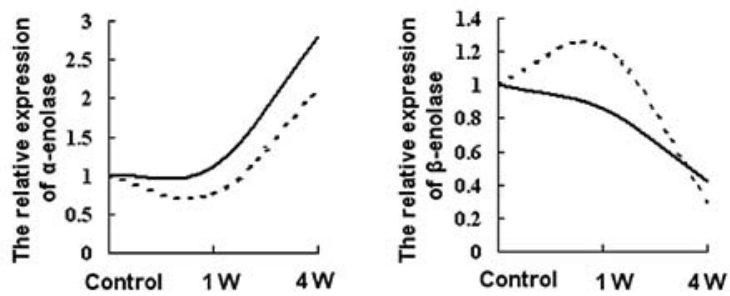

$1 \mathrm{~W}$
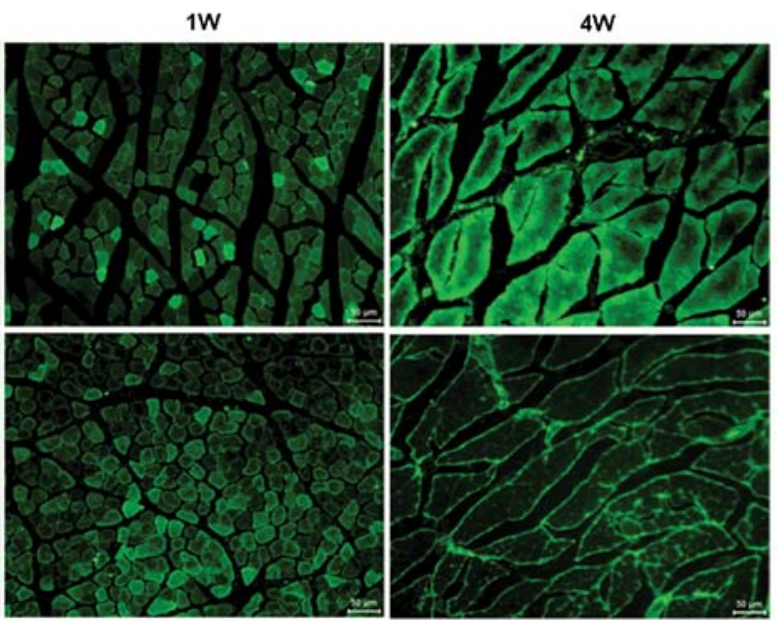

Figure 4. (A) The expression of $\alpha$ - and $\beta$-enolase in denervated tibialis anterior (TA) muscle at different time points post-nerve transaction was determined by western blot analysis. In curve plots, solid and dotted lines represent the expression change trend determined by western blot and iTRAQ-based analyses, respectively. (B) Immunofluorescence micrograph showing the $\alpha$ - and $\beta$-enolase expression changes in TA muscle during denervation-induced atrophy. 
A

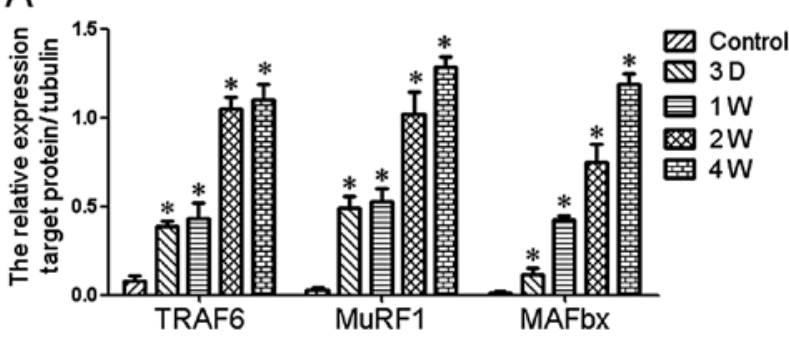

B

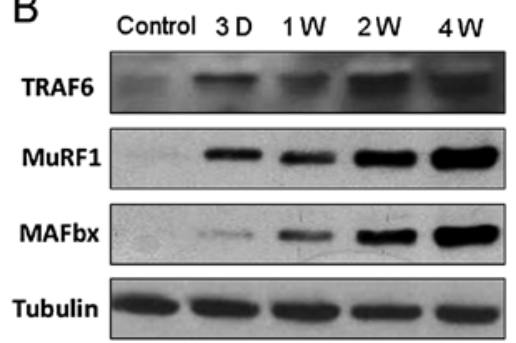

Figure 5. (A) Histograms comparing the expression levels of TRAF6, MuRF1, and MAFBx in tibialis anterior (TA) muscle at designated time points following sciatic nerve transection. "P<0.05 vs. control; D, days; W, weeks. (B) Representative western blot images, with tubulin serving as an internal control.

A

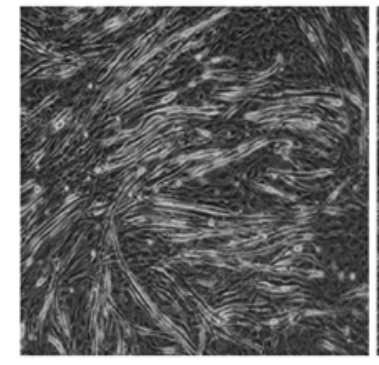

SIRNA-TRAF6

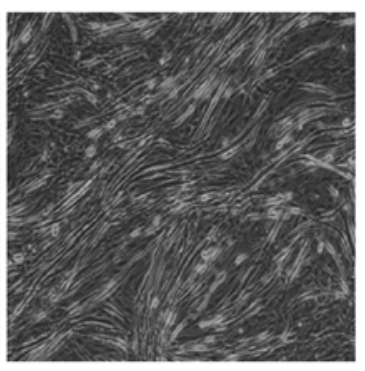

siRNA-control

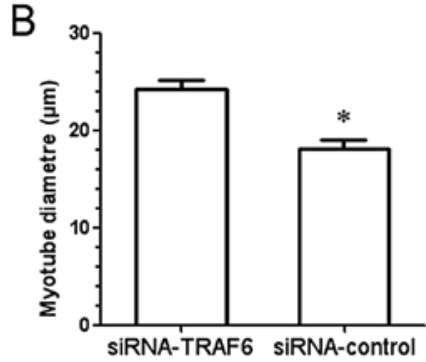

DEX-treated L6 myotube

C
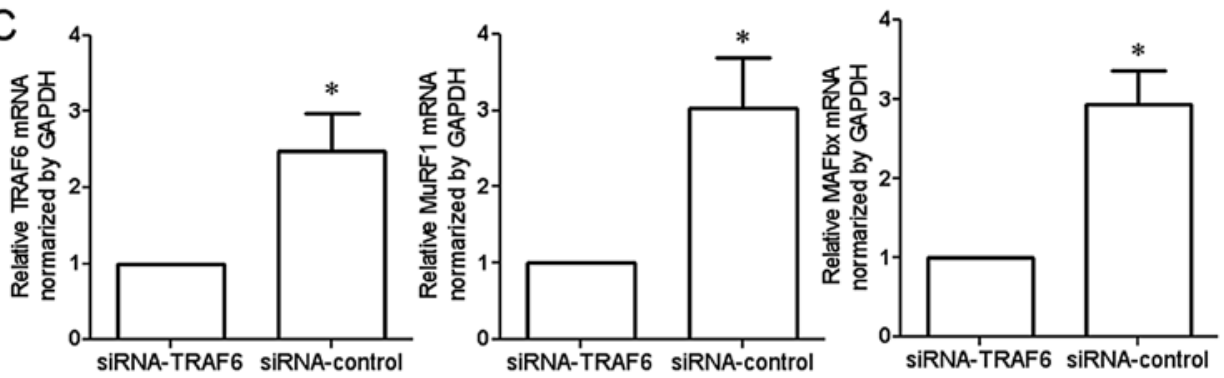

DEX-treated L6 myotube

Figure 6. Changes in dexamethasone (Dex)-treated L6 myotubes transfected with the small interfering RNA (siRNA) targeting TRAF6 and the negative siRNA control. (A) Light micrographs comparing the morphology of the myotubes. Scale bar, $50 \mu \mathrm{m}$. (B) Histogram showing the diameter of myotubes. (C) Histogram showing the mRNA expression of genes coding for TRAF6, MuRF1, and MAFBx, expressed relative to the expression of glyceraldehyde 3-phosphate dehydrogenase (GAPDH) ${ }^{*} \mathrm{P}<0.05$ vs. myotubes transfected with the TRAF6 siRNA.

energy metabolism-related enzymes are involved in the glycolytic, Krebs cycle and oxidative phosphorylation pathways. These observations suggest that time-dependent changes in energy production might be a dominant molecular event occurring in denervated skeletal muscle. The altered expression of energy metabolism-related proteins can lead to an overall disturbance of the muscle, and ultimately contribute to the establishment of pathological states, such as atrophy (20-22).

Enolase (2-phospho-D-glycerate hydrolase) is an essential dimeric glycolytic enzyme, and skeletal muscles contain 2 isoforms, $\alpha$ and $\beta(15,23)$. A previous study demonstrated that the ubiquitous $\alpha$-enolase and the muscle-specific $\beta$-enolase have the highest and lowest expression in undifferentiated myoblasts, respectively, and that a significant increase in the expression of $\beta$-enolase occurs upon differentiation of myoblasts and is maintained until the postnatal period (24). By contrast, $\alpha$-enolase has been rarely found expressed in the adult skeletal muscle. An isozymic switch from the embryonic $\alpha$ - towards the muscle- specific $\beta$-enolase has been observed during differentiation and maturation of myoblasts with high-energy requirements $(24,25)$. In our study, the expression of $\alpha$ - and $\beta$-enolase following muscle denervation in adult TA muscle was increased and decreased, respectively. This result suggests that an isozymic switch opposite to that occurring in muscle maturation (from the muscle-specific $\beta$ - towards the embryonic $\alpha$-enolase) takes place during denervation-induced muscle atrophy.

Enolase is a glycolytic enzyme that catalyses the conversion of 2-phosphoglycerate (2-PGA) to phosphoenolpyruvate (PEP) (24). $\beta$-Enolase binds with high affinity to sarcomeric troponin at the subcellular site where glycolysis-produced ATP is most needed for muscle contraction (26). In human muscles, the $\beta$-enolase subunit accounts for $>90 \%$ of the total enolase activity (27), and high levels of $\beta$-enolase characterize the glycolytic fast-twitch fibers of adult muscles. During the degeneration of myofibers, the drop in total enolase activity, mainly caused by a rapid decrease of $\beta$-enolase, correlates 
with myofiber degeneration (25). In this study, $\beta$-enolase was significantly downregulated in denervated TA muscle, which might relate to the reduced production of ATP in glycolysis, the failure in maintenance of the fast-twitch skeletal muscle phenotype, and the myofiber degeneration observed in atrophy. These hypotheses remain to be further investigated.

Enolase is well known as an enzyme of the glycolytic pathway, ubiquitously expressed in the cytosol of prokaryotic and eukaryotic cells (28). $\alpha$-enolase is however a multifunctional protein; in addition to its glycolytic activity, this protein has plasminogen receptor functions and plays a regulatory role in extracellular remodelling processes such as myogenesis $(29,30)$. In this study, $\alpha$-enolase was significantly upregulated in denervated TA muscle, which might be associated with early stages of myogenesis following nerve transection. Furthermore, $\alpha$-enolase was reported to be upregulated by hypoxia (31) and pro-inflammatory stimuli (32), which are two common pathogenic characteristics of muscle atrophy (33-36). Based on these reports, we hypothesize that the significant upregulation of $\alpha$-enolase might relate to the inflammatory environment of the denervated skeletal muscle. Pro-inflammatory cytokines play a key role in the pathophysiology of muscle atrophy through activation of atrophy-related genes, such as nucleasr factor (NF)- $\mathrm{B}$, TRAF6, MuRF1 and MAFbx (37-39). Therefore, it appears that $\alpha$-enolase plays a complex role in the regulation of denervation-induced muscle atrophy. The exact underlying mechanisms will be evaluated in a future study.

In proteomic studies, low-abundance proteins are commonly undetected. In this study, proteomic analysis indicated that the E2 polyubiquitin-conjugating enzyme UBE2N, required for TRAF6 activation (17), is upregulated in denervated TA muscle. This result, combined with predictions of proteinprotein interactions based on the STRING database, allowed us to infer that TRAF6 might be upregulated in denervated TA muscle, although the expression of this protein was not directly assessed by iTRAQ-based proteomics (undetected protein). This hypothesis was confirmed by western blotting. Our finding on TRAF6 expression in denervation-induced muscle atrophy is consistent with a previous study of starvation-induced muscle atrophy (18).

TRAF6 is a unique E3 ubiquitin ligase and adaptor protein involved in receptor-mediated activation of a number of signaling pathways, and its expression is enhanced during skeletal muscle atrophy (40). Deletion of the TRAF6 reduced the expression of the muscle-specific ubiquitin ligases MuRF1 and MAFBx (18), which are critical proteins in the development of muscle atrophy $(41,42)$. We further inferred that MAFBx and MuRF1 may be upregulated in denervated TA muscle. The inference was also confirmed by western blot analysis.

We hypothesized that the increased expression of $\alpha$-enolase in denervated TA muscle may relate to the activation of atrophy-related genes, including TRAF6, MuRF1 and MAFBx. Although these proteins were not identified by our proteomics analysis (possibly due to their low abundance), predicted interaction data from the STRING database allowed to infer a potential expression of the protein TRAF6 in denervated TA muscle. The expression of TRAF6, but also, MuRF1 and MAFBx proteins, was positively detected in denervated TA muscle with western blot analysis.
In order to investigate the potential involvement and the functional role of TRAF6 in the development of myotube atrophy, in this study, we examined the effects of siRNAmediated TRAF6 knockdown on dexamethasone-induced L6 myotube atrophy. In addition, the mRNA levels of TRAF6, MuRF1 and MAFBx were quantified by qRT-PCR in atrophied myotubes with the TRAF6 knockdown, and the results confirmed that TRAF6 may possibly exert its function through at least in part, regulating the muscle-specific ubiquitin ligases MAFBx and MuRF1.

In summary, the combined use of proteomics and bioinformatics provided additional knowledge on denervation-induced skeletal muscle atrophy. Hopefully, our findings may contribute to the understanding and treatment of skeletal muscle atrophy. This study also provided an example where a high number of proteins with high- or medium-abundance were identified with high confidence by an advanced proteomics technique, although even higher-sensitivity methods still remain to be developed so as to allow detection of low-abundance proteins. We suggest that subcellular fractionation techniques may be used in the future to reduce the sampling complexity and enrich for proteins of interest in skeletal muscle extracts, thereby allowing more a thorough analysis of the proteomic content of atrophied muscle.

\section{Acknowledgements}

This study was funded by the Hi-Tech Research and Development Program of China (863 Program, grant no. 2012AA020502), the National Key Basic Research Program of China (973 Program, grant nos. 2014CB542202 and 2014CB542203), the National Natural Science Foundation of China (grant nos. 81130080, 81171180,81301628 and 81073079), a project funded by the Priority Academic Program Development of Jiangsu Higher Education Institutions (PAPD), the Basic Research Project of the Jiangsu Education Department (grant no. 12KJB310010), the Colleges and Universities in Jiangsu Province graduate research project (grant no. CXZZ12_0861) and the Nantong Science and Technology Innovation Program (grant no. BK2011045). We thank Professor Jie Liu for assistance in the manuscript preparation.

\section{References}

1. Jackman RW and Kandarian SC: Am J Physiol Cell Physiol 287: C834-843, 2004.

2. Ibebunjo C, Chick JM, Kendall T, et al: Genomic and proteomic profiling reveals reduced mitochondrial function and disruption of the neuromuscular junction driving rat sarcopenia. Mol Cell Biol 33: 194-212, 2013.

3. McKinnell IW and Rudnicki MA: Molecular mechanisms of muscle atrophy. Cell 119: 907-910, 2004.

4. Romanick M, Thompson LV and Brown-Borg HM: Murine models of atrophy, cachexia, and sarcopenia in skeletal muscle. Biochim Biophys Acta 1832: 1410-1420, 2013.

5. Wei B, Dui W, Liu D, et al: MST1, a key player, in enhancing fast skeletal muscle atrophy. BMC Biology 11: 12, 2013.

6. Nagpal P, Plant PJ, Correa J, et al: The ubiquitin ligase Nedd4-1 participates in denervation-induced skeletal muscle atrophy in mice. PloS One 7: e46427, 2012.

7. Nader GA: Molecular determinants of skeletal muscle mass: getting the 'AKT' together. Int J Biochem Cell Biol 37: 1985-1996, 2005.

8. Ramamoorthy S, Donohue M and Buck M: Decreased Jun-D and myogenin expression in muscle wasting of human cachexia. Am J Physiol Endocrinol Metab 297: E392-401, 2009. 
9. Menconi MJ, Arany ZP, Alamdari N, et al: Sepsis and glucocorticoids downregulate the expression of the nuclear cofactor PGC-1beta in skeletal muscle. Am J Physiol Endocrinol Metab 299: E533-543, 2010.

10. Tews DS, Behrhof W and Schindler S: Expression patterns of initiator and effector caspases in denervated human skeletal muscle. Muscle Nerve 31: 175-181, 2005.

11. Bantscheff M, Boesche M, Eberhard D, et al: Robust and sensitive iTRAQ quantification on an LTQ Orbitrap mass spectrometer. Mol Cell Proteomics 7: 1702-1713, 2008.

12. Sun H, Li M, Gong L, et al: iTRAQ-coupled 2D LC-MS/MS analysis on differentially expressed proteins in denervated tibialis anterior muscle of Rattus norvegicus. Mol Cell Biochem 364 193-207, 2012.

13. Sinclair J, Metodieva G, Dafou D, et al: Profiling signatures of ovarian cancer tumour suppression using 2D-DIGE and 2D-LC-MS/MS with tandem mass tagging. J Proteomics 74 451-465, 2011

14. Szklarczyk D, Franceschini A, Kuhn M, et al: The STRING database in 2011: functional interaction networks of proteins, globally integrated and scored. Nucleic Acids Res 39: D561-D568, 2011.

15. Sun H, Zhu T, Ding F, et al: Proteomic studies of rat tibialis anterior muscle during postnatal growth and development. Mol Cell Biochem 332: 161-171, 2009.

16. Menconi M, Gonnella P, Petkova V, et al: Dexamethasone and corticosterone induce similar, but not identical, muscle wasting responses in cultured L6 and $\mathrm{C} 2 \mathrm{C} 12$ myotubes. J Cell Biochem 105: 353-364, 2008

17. Sanada T, Kim M, Mimuro H, et al: The Shigella flexneri effector OspI deamidates UBC13 to dampen the inflammatory response. Nature 483: 623-626, 2012.

18. Paul PK, Bhatnagar S, Mishra V, et al: The E3 ubiquitin ligase TRAF6 intercedes in starvation-induced skeletal muscle atrophy through multiple mechanisms. Mol Cell Biol 32: 1248-1259, 2012.

19. Jones A, Hwang DJ, Narayanan R, et al: Effects of a novel selective androgen receptor modulator on dexamethasone-induced and hypogonadism-induced muscle atrophy. Endocrinology 151: 3706-3719, 2010.

20. Wang X, Pickrell AM, Rossi SG, et al: Transient systemic mtDNA damage leads to muscle wasting by reducing the satellite cells pool. Hum Mol Genet 22: 3976-3986, 2013.

21. Calvani R, Joseph AM, Adhihetty PJ, et al: Mitochondrial pathways in sarcopenia of aging and disuse muscle atrophy. Bio Chem 394: 393-414, 2013.

22. Picard M, Ritchie D, Thomas MM, et al: Alterations in intrinsic mitochondrial function with aging are fiber type-specific and do not explain differential atrophy between muscles. Aging Cell 10: 1047-1055, 2011.

23. Sun H, Liu J, Ding F, et al: Investigation of differentially expressed proteins in rat gastrocnemius muscle during denervation-reinnervation. J Muscle Res Cell Motil 27: 241-250, 2006.

24. Keller A, Peltzer J, Carpentier G, et al: Interactions of enolase isoforms with tubulin and microtubules during myogenesis. Biochim Biophys Acta 1770: 919-926, 2007.

25. Merkulova T, Dehaupas M, Nevers MC, et al: Differential modulation of alpha, beta and gamma enolase isoforms in regenerating mouse skeletal muscle. Eur J Biochem 267: 3735-3743, 2000.
26. Merkulova T, Lucas M, Jabet C, et al: Biochemical characterization of the mouse muscle-specific enolase: developmental changes in electrophoretic variants and selective binding to other proteins. Biochem J 323 (Pt 3): 791-800, 1997.

27. Comi GP, Fortunato F, Lucchiari S, et al: Beta-enolase deficiency, a new metabolic myopathy of distal glycolysis. Ann Neurol 50: 202-207, 2001

28. Seweryn E, Pietkiewicz J, Szamborska A, et al: Enolase on the surface of prokaryotic and eukaryotic cells is a receptor for human plasminogen. Postepy Hig Med Dosw (Online) 61: 672-682, 2007 (In Polish).

29. Díaz-Ramos A, Roig-Borrellas A, García-Melero A, et al: Requirement of plasminogen binding to its cell-surface receptor alpha-enolase for efficient regeneration of normal and dystrophic skeletal muscle. PloS One 7: e50477, 2012.

30. Díaz-Ramos A, Roig-Borrellas A, García-Melero A, et al: alphaEnolase, a multifunctional protein: its role on pathophysiological situations. J Biomed Biotechnology 2012: 156795, 2012.

31. Aaronson RM, Graven KK, Tucci M, et al: Non-neuronal enolase is an endothelial hypoxic stress protein. J Biol Chem 270: 27752-27757, 1995.

32. Fontán PA, Pancholi V, Nociari MM, et al: Antibodies to streptococcal surface enolase react with human $\alpha$-enolase: implications in poststreptococcal sequelae. J Infect Dis 182: 1712-1721, 2000.

33. Paul PK, Gupta SK, Bhatnagar S, et al: Targeted ablation of TRAF6 inhibits skeletal muscle wasting in mice. J Cell Biol 191: 1395-1411, 2010

34. Zhao W, Pan J, Zhao Z, et al: Testosterone protects against dexamethasone-induced muscle atrophy, protein degradation and MAFbx upregulation. J Steroid Biochem Mol Biol 110: 125-129, 2008.

35. Castillero E, Alamdari N, Lecker SH, et al: Suppression of atrogin-1 and MuRF1 prevents dexamethasone-induced atrophy of cultured myotubes. Metabolism 62: 1495-1502, 2013.

36. Castillero E, Alamdari N, Aversa Z, et al: PPAR $\beta / \delta$ regulates glucocorticoid- and sepsis-induced FOXO1 activation and muscle wasting. PloS One 8: e59726, 2013.

37. Suetta C, Frandsen U, Jensen L, et al: Aging affects the transcriptional regulation of human skeletal muscle disuse atrophy. PloS One 7: e51238, 2012

38. Caron AZ, Haroun S, Leblanc E, et al: The proteasome inhibitor MG132 reduces immobilization-induced skeletal muscle atrophy in mice. BMC Musculoskelet Disord 12: 185, 2011.

39. Caron AZ, Drouin G, Desrosiers J, et al: A novel hindlimb immobilization procedure for studying skeletal muscle atrophy and recovery in mouse. J Appl Physiol 106: 2049-2059, 2009.

40. Paul PK and Kumar A: TRAF6 coordinates the activation of autophagy and ubiquitin-proteasome systems in atrophying skeletal muscle. Autophagy 7: 555-556, 2011.

41. Cao PR, Kim HJ and Lecker SH: Ubiquitin-protein ligases in muscle wasting. Int J Biochem Cell Biol 37: 2088-2097, 2005.

42. Zhang L, Tang H, Kou Y, et al: MG132-mediated inhibition of the ubiquitin-proteasome pathway ameliorates cancer cachexia. J Cancer Res Clin Oncol 139: 1105-1115, 2013. 